\title{
Heart Failure and Stress Response
}

\section{Alessandro Pingitore, Francesca Mastorci, Giorgio lervasi}

National Research Council, Institute of Clinical Physiology, Pisa, Italy

http://www.ifc.cnr.it/index.php/en/

ONLINE: $\quad 22$ Dec 2015

KEYWORDS: heart failure, open access, cardiology research.

With the current special issue on HEart FAilure and STress Response (HEFAST), the Biomedical Data Journal (BMDJ) aims to introduce a holistic approach to the publication, sharing, linking, review and evaluation of heart failure (HF) studies, based on the open access to scientific information.

In cardiology research this is critical in order to promote interoperability between different centres through integration of published biomedical datasets. In particular, this can serve as a source for simulation and integration of computational technologies of diseases and biological processes. It is increasingly recognized that the integration of a variety of biological and medical research data to produce or refine disease models using advanced statistical, computational and mathematical approaches could facilitate the understanding of biological systems' complexity.

To reflect on these developments, publication of datasets on experimental in vivo and ex vivo models of $\mathrm{HF}$ and on patients with chronic ischemic and non-ischemic HF can be applied to study development of diseases via systems medicine. Heart failure starts as single-organ disease and advances as a systemic disease during its evolution in which the dysfunction of other organs has a relevant clinical and prognostic impact. ${ }^{1}$ In a pathophysiology view, HF can be seen in a unique scenario of altered systemic homeostasis, in which heart and peripheral organ dysfunction, derangement of the neuroendocrine and immune systems represent chronic stress stimuli, with continuous activation of stress mechanisms. This response, defined by McEwen as allostatic load, which can evolve into allostatic overload as an extreme form of allostasis, is the price the body pays for being continuously forced to adapt to adverse physical and pathophysiological conditions. ${ }^{2,3}$

Considering the intriguing model of $\mathrm{HF}$ as chronic disease, experimental (animal datasets) and clinical observations (human datasets), could help to address the current knowledge gaps in disease pathophysiology in order to support innovation in the development of novel, evidence-based treatments.

The previous issue of the Biomedical Data Journal already presented datasets, related to the subject of heart failure. Bunevicius et al published data collected with the aim to foresee functional and cognitive outcomes of patients with ischemic stroke, ${ }^{4}$ while Stropute with colleagues described data used to identify Type $D$ (distressed) personality in Lithuanian patients with coronary artery disease. ${ }^{5}$ 
The papers in this issue, divided in experimental and clinical articles, as well as the respective datasets, also adhere to highest standards of "gold" open access.

The first group of papers analyses the need for open access, structured data of confirmed quality in the field of cardiac remodelling after myocardial infarction (MI) in rats and mice. ${ }^{6,7}$ The publication of well-structured data from molecular and cellular levels and translated towards to the heart could provide the basis for the development of a new approach to integrate different advances into a unified model of heart function and disease. In addition, datasets presented in this first part referring to the experimental studies, could be valuable tools in understanding the adaptation of the heart to stress through the development of computational models. Modelling the response of the heart after $\mathrm{MI}$ could predict recovery of function or development of HF and can bridge the gap between preclinical and clinical setting by integrating data obtained from experimental cell and animal based models to patients.

The third article examines the development of a modelling platform composed by several modules that can be assembled and used according to the specific need, with focus on systemic and pulmonary circulation, models of congenital heart defects, models of heart assist devices, and of autonomic controls. ${ }^{8}$ This platform was applied to different clinical problems that have in common the use of the model to analyse complex pathophysiological situations and evaluate the effects of different approaches to the problem. The application of this model permits to reproduce patient's status and simulate, starting from this point, the effects of different clinical choices.

The final section looks at clinical aspects of HF, focused on the interplay between biochemical parameters and cardiac function. Vassalle et al. evaluated the prognostic value of anaemia in patients with chronic HF; it was associated with inflammatory and oxidative stress indices and can represent an independent predictor of mortality. ${ }^{9}$ Di Bella et al. present datasets in cardiac magnetic resonance (CMR) obtained from a wide range of patients with previous $\mathrm{MI}$, thus providing an opportunity to approach cardiac research studies in an integrated, multidisciplinary and systematic framework, where clinical dataset can be reused to assess the CMR role in the prognostic stratification of patients with previous $\mathrm{MI}^{10}$

In conclusion, data sharing of experimental, clinical, bio-humoral, and imaging variables could allow generation of a multiparametric and integrated approach to identify and characterize HF patients in a new and intriguing ways.

\section{References}

1. Sabatino L, lervasi G, Pingitore A. Thyroid Hormone and Heart Failure: From Myocardial Protection to Systemic Regulation. Expert Rev Cardiovasc Ther. 2014;12(10):1227-36. doi: 10.1586/ 14779072.2014.957674.

2. Karatsoreos IN, McEwen BS. Psychobiological allostasis: resistance, resilience and vulnerability. Trends Cogn Sci. 2011;15(12):576-84. doi: 10.1016/j.tics.2011.10.005.

3. Peters A, McEwen BS. Stress habituation, body shape and cardiovascular mortality. Neurosci Biobehav Rev. 2015;56:139-50. doi: 10.1016/j.neubiorev.2015.07.001.

4. Bunevicius A, Kazlauskas H, Raskauskiene N, Janusonis V, Bunevicius R. Thyroid Hormone and CReactive Protein Serum Concentrations, Disease Severity and Discharge Outcomes of Ischemic Stroke Patients: A Dataset. Biomed Data J. 2015;1(2):13-18. doi: 10.11610/bmdj.01204.

5. Stropute D, Bunevicius A, Staniute M, Brozaitiene J, Bunevicius R. Type D (Distressed) Personality in Lithuanian Patients with Coronary Artery Disease: A Dataset. Biomed Data J. 2015;1(2):26-30. doi: 10.11610/bmdj.01205.

6. Mourouzis I, Mantzouratou P, Pantos C. A Dataset from Echocardiographic Assessment of Cardiac Function in Mice after Myocardial Infarction. Biomed Data J. 2015;1(3):37-40. doi: 10.11610/ bmdj.01301. 
7. Mourouzis I, Pantos C. Echocardiographic Datasets Showing Development of Cardiac Remodelling in Rats at Different Time-points after Acute Myocardial Infarction. Biomed Data J. 2015;1(3):41-44. doi: $10.11610 /$ bmdj.01302.

8. Ferrari G, Di Molfetta A, Zieliński K, Fresiello L. Circulatory Modelling as a Clinical Decision Support and an Educational Tool. Biomed Data J. 2015;1(3):45-50. doi: 10.11610/bmdj.01303.

9. Vassalle C, Vannucci A, Mastorci F, Battaglia D, Landi P, Di Cecco P, Carpeggiani C. Anaemia as Predictor of All-Cause Mortality in Patients with Systolic Dysfunction. Biomed Data J. 2015;1(3):5155. doi: 10.11610/bmdj.01304.

10. Di Bella G, Aquaro G, Mastorci F, Pingitore A. Prognostic Role of Cardiac Magnetic Resonance Imaging in Patients with Previous Myocardial Infarction: A Clinical and Imaging Dataset. Biomed Data J. 2015;1(3):56-60. doi: 10.11610/bmdj.01305. 